\title{
FEAR ACQUISITION AND GENERALIZATION IN
}

\section{GENERALIZED ANXIETY DISORDER}

Daniella Tinoco-González, ${ }^{1}$ Miquel Angel Fullana,,${ }^{1,2, *}$ David Torrents-Rodas, ${ }^{1}$ Albert Bonillo, ${ }^{3}$ Bram Vervliet, ${ }^{4}$ María Jesús Blasco, ${ }^{2}$ Magí Farré, ${ }^{5}$ and Rafael Torrubia ${ }^{1}$

${ }^{1}$ Department of Psychiatry and Forensic Medicine, Institute of Neurosciences, School of Medicine, Universitat Autònoma de Barcelona, Bellaterra, Spain

${ }^{2}$ Anxiety Unit, Institute of Neuropsychiatry and Addictions (INAD), Hospital del Mar, Parc de Salut MAR, Barcelona, Spain

${ }^{3}$ Department of Psychobiology and Methodology of Health Sciences, School of Psychology, Universitat Autònoma de Barcelona, Bellaterra, Spain

${ }^{4}$ Center for Excellence on Generalization in Health and Psychopathology, KU Leuven University, Leuven, Belgium

${ }^{5}$ Human Pharmacology and Clinical Neurosciences Research Group, Neuroscience Research Program, Hospital del Mar Research Institute (IMIM), Parc de Salut MAR, Universitat Autònoma de Barcelona, Bellaterra, Spain

\section{*CORRESPONDING AUTHOR \& ADDRESS}

Miquel A. Fullana

Institute of Neuropsychiatry \& Addictions (INAD), Hospital del Mar.

Passeig Marítim, 25/29. 08003 Barcelona, Spain.

E-mail address: miguelangelfullana@gmail.com

Phone number: (0034) 93248346

\section{Acknowledgements}

We thank Shmuel Lissek, Ph.D., for lending us his paradigm, as well as for his general advice for the implementation of the paradigm in our laboratory. This research was financially supported by the Ministerio de Ciencia e Innovación, Spanish government (PS09/00307) and by the Comissionat per a Universitats i Recerca, Generalitat de

Catalunya (2009SGR51). D. T-G. and D. T-R. are recipients of a Ph.D. fellowship from the Generalitat de Catalunya (FI2010). 


\begin{abstract}
Abnormal fear conditioning processes (including fear acquisition and conditioned fear-generalization) have been implicated in the pathogenesis of anxiety disorders. Previous research has shown that individuals with panic disorder present enhanced conditioned fear-generalization in comparison to healthy controls. Enhanced conditioned fear-generalization could also characterize generalized anxiety disorder (GAD), but research so far is inconclusive. An important confounding factor in previous research is comorbidity. The present study examined conditioned fear-acquisition and fear-generalization in 28 patients with GAD and 30 healthy controls using a recently developed fear acquisition and generalization paradigm assessing fear-potentiated startle and online expectancies of the unconditioned stimulus. Analyses focused on GAD patients without comorbidity but included also patients with comorbid anxiety disorders. Patients and controls did not differ as regards fear acquisition. However, contrary to our hypothesis, both groups did not differ either in most indexes of conditioned fear-generalization. Moreover, dimensional measures of GAD symptoms were not correlated with conditioned fear-generalization indexes. Similar results were obtained for patients with comorbidity. These results suggest that conditioned fear-generalization is not enhanced in GAD. Results are discussed with special attention to the possible effects of comorbidity on fear learning abnormalities.
\end{abstract}

\title{
Keywords
}

Fear conditioning, fear generalization, generalized anxiety disorder. 


\section{Introduction}

Fear conditioning is a form of associative learning by which a neutral stimulus is repeatedly paired with an aversive unconditioned stimulus (US), becoming a conditioned stimulus (CS), which is capable of eliciting a conditioned fear response (CR). Although fear conditioning is an adaptive form of learning, it may become a source of pathology when anxious reactivity to a CS persists in the absence of a CS/US association.

Several fear conditioning processes have been implicated in the pathogenesis of anxiety disorders (Lissek et al., 2005; Mineka \& Zinbarg, 2006). These processes include acquisition, within-session extinction, extinction recall, conditioned inhibition and conditioned fear-generalization. For example, an enhanced fear acquisition may be characteristic of social phobia (Lissek et al., 2008a), generalized anxiety disorder (GAD; Thayer, Friedman, Borkovec, Johnsen, \& Molina, 2000), or post-traumatic stress disorder (PTSD; Orr et al., 2000; Peri, Ben-Shakhar, Orr, \& Shalev, 2000). Impaired within-session fear extinction has been shown for individuals with panic disorder (PD; Michael, Blechert, Vriends, Margraf, \& Wilhelm, 2007; Otto et al., 2014) or GAD (Pitman \& Orr, 1986), whereas impaired extinction recall could be present in PTSD (Milad et al., 2008) or obsessive-compulsive disorder (OCD; Milad et al., 2013). Moreover, conditioned inhibition deficits may characterize PTSD (Jovanovic et al., 2009, 2010). However, these results have not been always replicated. For example, "normal" (i.e. not enhanced) fear acquisition has been reported in PD (Michael et al., 2007), social phobia (Tinoco-González et al., 2013; Hermann, Ziegler, Birbaumer, \& Flor 2002) or GAD (Pitman \& Orr, 1986).

Conditioned fear-generalization occurs when fear CRs extend to a range of novel 
stimuli (generalization stimuli) that resemble the original CS. It can become maladaptive (i.e. excessive) when some of these stimuli are perceived as harmful given the similarity and (non)discriminability to previously learned stimuli (Dunsmoor, Mitroff, \& La Bar, 2009).

The possible role of conditioned fear-generalization in pathological anxiety has gained increased research recognition during the last decade (Vervliet, Vansteenwegen, \& Eelen, 2004; Vervliet, Vansteenwegen, Baeyens, Hermans, \& Eelen, 2005; Lissek et al., 2008b, 2010, 2013; Hajcak et al., 2009). Indeed, recent etiological accounts of anxiety disorders suggest that conditioned fear-generalization could be a central pathogenic marker of some anxiety disorders (Lissek, 2012), although prospective studies supporting this assumption are lacking. In several case-control studies, individuals with specific anxiety disorders have shown abnormal (i.e. enhanced) conditioned fear- generalization in comparison to healthy controls. This has been the case for PD (Lissek et al., 2010), PTSD (cited in Lissek et al., 2008b), and recently, GAD (Lissek et al., 2013). In GAD, generalization may contribute to an increase in the number of events triggering worry, the hallmark of the disorder (Greenberg, Carlson, Cha, Hajcak, \& Mujica-Parodi, 2013). It may also contribute to worry about topics that only have a moderate relatedness with the original triggers (Lissek et al., 2013).

In the aforementioned study, Lissek et al. (2013) compared fear acquisition and conditioned fear-generalization using a validated experimental paradigm (Lissek et al., 2008b) among 22 patients with GAD and 26 healthy controls. GAD patients showed abnormally broad conditioned fear-generalization gradients, compared to controls, as measured by the fear-potentiated startle, despite showing similar fear acquisition. However, in a recent study no evidence for enhanced conditioned fear-generalization in subjective 
(risk ratings) or autonomic (pupillary response) measures was found in women with GAD in comparison to healthy controls (Greenberg et al., 2013). A limitation of these two studies on conditioned fear-generalization in GAD is that almost $50 \%$ of patients had a comorbid anxiety (Lissek et al., 2013) or depressive (Greenberg et al., 2013) disorder. This casts doubts about the specificity of conditioned fear-generalization impairments in GAD. Additionally, studies on the role of certain individual differences variables that are closely related to GAD (e.g., trait-anxiety or trait-worry) in non-clinical or sub-clinical individuals using fear conditioning paradigms have also provided inconsistent results. For example, high-trait anxiety has been associated with enhanced fear acquisition in some studies (Indovina, Robbins, Núñez-Elizalde, Dunn, \& Bishop, 2011), but not in others (Torrents-Rodas et al., 2013). The same is true for trait-worry (Joos, Vansteenwegen, \& Hermans, 2012; Otto et al., 2007). These studies used relatively different conditioning tasks and a possible explanation for the inconsistent results is that ambiguous tasks may obscure the effects of trait anxiety (Torrents-Rodas et al, 2012; see Beckers, Krypotos, Boddez, Effting, \& Kindt, 2013).

Although fear conditioning processes do not feature prominently among current theoretical explanations of GAD (see Behar, DiMarco, Hekler, Mohlman, \& Staples 2009; Newman, Llera, Erickson, Przeworski, \& Castonguay, 2013), some typical GAD characteristics such as the tendency to interpret ambiguous stimuli as threatening (see Lissek et al., 2013) suggest that these processes may have an important role in the disorder and that more studies in the field are needed. In the present study, we examined conditioned fear-acquisition and fear-generalization in GAD patients using a fear conditioning paradigm assessing fear-potentiated startle (FPS) and subjective responses. To address the issue of comorbidity, we focused our analyses on GAD participants without comorbidity but 
conducted additional separate analyses in GAD patients with comorbid anxiety disorders. Based on previous research, we predicted that GAD patients would show enhanced conditioned fear-generalization in comparison to healthy controls (Lissek et al., 2013) but we expected that GAD patients and healthy controls would show similar fear acquisition (Lissek et al., 2013).

Method

\section{Participants and procedure}

Participants were recruited among university students by advertisements to participate in a study on "physical responses to emotions". We did not rely on clinical referral to facilitate the recruitment of unmedicated GAD participants. Initially, 2005 individuals were screened with the Spanish version of the Carroll-Davidson screening scale for DSM-IV Generalized Anxiety Disorder (CD-GAD scale; Bobes, García-Calvo, Prieto, García-García, \& Rico-Villademor, 2006) via a secure web system. Those scoring high ( $>6$; percentile 74) or low $(<3$; percentile 37$)$ on the CD-GAD scale were offered to participate in the study and, if accepted, were interviewed by a licensed clinical psychologist not involved in the experimental phase using the Mini International Neuropsychiatric Interview (MINI, Sheehan et al., 1998).

Exclusion criteria for all participants were 1) pharmacological medication or medical pathology (e.g. neurological disorders) capable of interfering with study objectives, 2) use of illicit drugs, 3) pregnancy, 4) psychomotor delay (item A3c of the MINI), and 5) not being Spanish-speaking. Additional exclusion criteria for GAD participants (also assessed with the MINI) were presence of current major depressive disorder or suicidal 
ideation and past history of major depressive disorder, bipolar disorder, or psychotic disorder.

The final sample consisted of 28 individuals ( $n=5$ with comorbid social anxiety disorder) who fulfilled current diagnostic criteria of GAD and 30 healthy controls (HC) who did not fulfill criteria for any present or past mental disorder. Healthy controls were distilled from the initial screening sample based on their GAD scale scores (GAD $<3)$ and optimal matching with regard to age and gender. Demographic and clinical characteristics of the participants are presented in Table 1.

\section{Measures}

Our screening instrument, the CD-GAD scale is a 12 -item scale that assesses GAD symptoms during the past 6 months based on DSM-IV-TR criteria. A score of 6 or above indicates a positive screen for GAD. The Spanish version of the CD-GAD (Bobes et al., 2006) has shown similar psychometric properties to the original version.

The MINI is a widely used structured psychiatric interview that assesses current and life-time mental disorders and has shown to have good concordance with other diagnostic measures, and good interrater and test-retest reliability (Sheehan et al., 1998; Lecubrier et al., 1997). The Spanish validated version of the MINI was used (Bobes, 1998).

During the experimental session, all participants completed the validated Spanish versions of the State-Trait Anxiety Inventory (STAI-S and STAI-T; Spielberger, Gorsuch \& Lushene, 1982), which measure state (i.e. current) and trait (i.e. dispositional) anxiety, and have sound psychometric properties (Spielberger, Gorsuch \& Lushene, 1982). The STAI-T has been used as an outcome measure in several treatment trials in GAD (Fisher \& Durham, 1999). 
Participants also completed the Spanish version (Sandín, Valiente, Chorot \& Santed, 2007) of the Anxiety Sensitivity Index-3, a measure of fear of anxiety symptoms with excellent psychometric properties (Sandín et al., 2007).

All participants were paid $15 €$ and were asked to sign an informed consent, which was previously approved by the corresponding institution's Ethical Research Committee, after describing the experimental procedure in detail.

\section{Table 1.}

Demographic and clinical characteristics of participants and variables related to the experimental procedure.

\begin{tabular}{|c|c|c|c|c|c|}
\hline \multicolumn{6}{|c|}{ Group } \\
\hline \multirow[b]{2}{*}{ Variable } & \multicolumn{2}{|c|}{$\begin{array}{l}\text { Non-comorbid GAD } \\
\text { Participants } \\
(n=23)\end{array}$} & \multicolumn{3}{|c|}{ Healthy Controls $(n=30)$} \\
\hline & Mean & $\mathrm{SD}$ & Mean & SD & Significance $^{\mathrm{a}}$ \\
\hline Age & 24.30 & 5.37 & 26.60 & 4.34 & $p=.10$ \\
\hline CD-GAD (0-12) & 9.27 & 2.03 & 0.63 & 1.25 & $p<.001$ \\
\hline ASI-3 (0-64) & 26.09 & 11.88 & 11.53 & 4.48 & $p<.001$ \\
\hline STAI-S $(0-60)$ & 17.02 & 8.69 & 9.27 & 5.01 & $p<.001$ \\
\hline STAI-T $^{\mathrm{b}}(0-60)$ & 36.65 & 9.49 & 10.30 & 6.50 & $p<.001$ \\
\hline Shock intensity (mA) & 3.71 & 1.00 & 3.96 & 1.07 & $p=.39$ \\
\hline Shock discomfort (1-10) & 5.17 & 2.27 & 4.67 & 2.01 & $p=.39$ \\
\hline \multirow[t]{2}{*}{ Startle probe discomfort (1-10) } & 7.78 & 1.45 & 6.70 & 1.88 & $p=.03$ \\
\hline & $\mathrm{N}$ & $\%$ & $\mathrm{~N}$ & $\%$ & \\
\hline Female Gender & 17 & 73.9 & 17 & 56.7 & $p=.19$ \\
\hline Contingency-unaware & 6 & 26.1 & 5 & 16.7 & $p=.40$ \\
\hline
\end{tabular}

CD-GAD, Carroll-Davidson screening scale for DSM-IV Generalized Anxiety Disorder; ASI, Anxiety

Sensitivity Index-3; STAI-S State-Trait Anxiety Inventory, State Version; STAI-T, State-Trait Anxiety Inventory, Trait Version; SD, Standard Deviation

${ }^{a}$ Independent sample $t$ tests (except for gender and awareness which were assessed with a Chi square)

${ }^{\mathrm{b}} \mathrm{Scores}$ range from 0 to 60 in the Spanish version of the STAI-S and STAI-T.

${ }^{1}$ The corresponding numbers for the GAD subjects with comorbidity ( $\left.\mathrm{n}=28\right)$ were Mean (SD): Age = 25.11 (6.39); CD-GAD = 9.36 (2.11); ASI-3 = 26.04 (11.77); STAI-S = 18.68 (9.11); STAI-T = 36.82 (8.89); Shock intensity = 3.75 (0.99); Shock discomfort = 5.21 (2.33); Startle probe discomfort $=7.71(1.56)$; Female gender $=71.4 \%$; Contingency-unaware $=25 \%$. 


\section{Stimuli and procedure}

We used the paradigm developed by Lissek and colleagues, which consists of three experimental phases (pre-acquisition, acquisition, and generalization) preceded by one habituation phase (Lissek et al., 2008b) and which allows the study of both conditioned fear acquisition and generalization.

Ten rings of gradually increasing size were presented for $8 \mathrm{~s}$ on a computer monitor and served as conditioned stimuli (CSs) and generalization stimuli (GSs). The diameter of the smallest ring was $5.08 \mathrm{~cm}$ and subsequent rings increased by $15 \%{ }^{2}$. The rings at the two extremes of this size continuum served as CSs. For half of the participants in each group, the smallest ring was the CS+ (paired with the US before its offset) and the largest was the CS-; for the remaining participants the pairing was reversed. The intermediate rings were used to test conditioned generalization. A fixation-cross (size: $8.23 \mathrm{~cm}$ ) appeared on the screen when no stimulus was presented (inter-trial interval, ITI). The US was an electric shock of $10 \mathrm{~ms}$ duration, with an intensity adjusted for each participant as being "highly uncomfortable but not painful", delivered to the volar surface of the right forearm. The acoustic startle probe was a $50 \mathrm{~ms}$ duration, $102 \mathrm{~dB}(\mathrm{~A})$ burst of white noise with a near instantaneous rise time, presented binaurally through headphones. Startle probes were presented 4 or $5 \mathrm{~s}$ after the beginning of odd trials, inter-probe intervals (IPIs) ranged from 18 to $25 \mathrm{~s}$. ITI durations ( 9 to $17 \mathrm{~s}$ ) were adjusted to keep IPIs within the specified range. During even trials, online ratings of perceived risk of shock for each stimulus were obtained $(1=$ no risk, $2=$ moderate risk, $3=$ high risk $)$. One or $2 \mathrm{~s}$ after trial onset, a

\footnotetext{
${ }^{2}$ As in Lissek et al., 2008b, the diameter for the smallest ring (Ring \#1) was 2.00 in and subsequent rings increased by $15 \%$ with Ring \#2 increasing 15\% from Ring \#1 (2.30 in), Ring \#3 increasing 30\% from Ring \#1 (2.60 in), Ring \#4 increasing $45 \%$ from \#1 (2.90 in), and
} 
question (Level of risk?) cued participants to respond as quickly as possible using a computer keyboard. Stimulus timing and response recording were controlled by the commercial system Presentation (Neurobehavioral Systems Inc, Version 0.70, www.neurobs.com).

Participants were not instructed about the CS-US contingency, but were told that they might learn to predict the shock if they attended to the presented stimuli. Then the electrodes were placed, and the intensity of the shock was adjusted. After placing the headphones, nine startle probes were presented to reduce initial startle reactivity (habituation). Pre-acquisition consisted of six CS+ and six CS- trials presented in the absence of the US. Acquisition consisted of $12 \mathrm{CS}+$ (with a 75\% reinforcement) and 12 CS- trials. Generalization consisted of 12 CS+ (with a 50\% reinforcement), 12 CS-, and six trials from each of the eight GSs sizes. Trials for all the phases were presented in quasi-random order with the restriction that no more than two stimuli of the same class appeared consecutively. Furthermore, to ensure an even distribution of trial types, the trials were arranged into two and six blocks for acquisition and generalization phases, respectively. In addition, an equal number of each trial type was used for the recording of psychophysiological measures (recorded in odd trials) and risk ratings (recorded in even trials). ITI trials were intermixed with CS and GSs trials across the experimental session (six in pre-acquisition, 12 in acquisition and generalization). In half of the ITI trials, startle probes were also presented. There was a 5 min break between the acquisition and generalization phases. Self-reported levels of anxiety and arousal evoked by CS+ and CSwere collected using 10-point Likert scale following acquisition and generalization phases.

so on. Such size increments resulted in ring diameters, from smallest to largest, of 2.00, 2.30, 2.60, 2.90, 3.20, 3.50, 3.80, 4.10, 4.40, and 4.70 in. 
Additionally, after the experiment, participants rated the discomfort produced both by the US and the startle probe on a 1 (no discomfort) to 10 (maximum discomfort) scale; and answered a multiple-choice question (based on Dawson \& Reardon, 1973) regarding contingency awareness ("The electric stimulus usually appeared: a) in the presence of the smallest ring; b) in the presence of the biggest ring; c) randomly; d) I don't know"). Individuals who correctly identified the stimulus that co-occurred with the US were considered contingency-aware.

\section{Physiological recordings}

Physiological responses were recorded using the BIOPAC Mod. MP150WSW recording system (Biopac Systems Inc., Santa Barbara, CA). The startle blink response was measured by recording the electromyographic activity (EMG) of the orbicularis oculi, using two $0.5 \mathrm{~cm} \mathrm{Ag} / \mathrm{AgCl}$ surface electrodes and following standard guidelines (Blumenthal et al., 2005). Impedance level was maintained below $5 \mathrm{k} \Omega$. The raw EMG signal was sampled at a rate of $2000 \mathrm{~Hz}$, filtered to reduce power line noise (analogue $50 \mathrm{~Hz}$ notch filter) and to attenuate the frequencies beyond the EMG spectrum (infinite impulse response band-pass filter, cut-off frequencies of 28 and $500 \mathrm{~Hz}$ ), and then rectified and smoothed off-line (10 ms moving window average), using the AcqKnowledge v.4.1 software (Biopac Systems, Inc, Santa Barbara, CA).

\section{Data reduction and response definition}

The onset latency window for the startle response was 20 to $100 \mathrm{~ms}$ and the peak magnitude was determined within $150 \mathrm{~ms}$ of response onset. Startle amplitudes were computed in microvolts $(\mu \mathrm{V})$ as the difference between the EMG value at response peak 
and the average EMG during the baseline period (50 ms preceding startle probe onset). In those trials in which no response was detected, amplitude was scored as $0 \mu \mathrm{V}$. After visual inspection, trials with excessive baseline activity were rejected. The number of rejected trials was not different between groups [GAD: $3.11 \%$, HC: $\left.2.65 \% ; \chi^{2}(1)=6.79, p>.05\right]$. Prior to statistical analysis, startle responses were T-transformed for each participant individually and for each phase separately. For online risk ratings, one GAD participant was excluded from the analysis due to technical problems.

\section{Data analysis}

Differences in variables related to the experimental procedure were assessed with $t$-tests. Preliminary analyses for overall startle reactivity during the whole experiment were

performed by repeated-measures ANOVA of startle responses during ITI trials (raw data). Factors were phase/block (pre-acquisition, first block of acquisition, second block of acquisition, and generalization) and group (GAD, HC). Additionally, a 2 (GAD, HC) x 9 (all 9 trials of the habituation phase) ANOVA was computed to analyze habituation to the startle probe before the experiment began.

Data were analyzed separately for (1) preacquisition and acquisition and (2) generalization, and for each measure (risk ratings and startle EMG), using repeated-measures ANOVAs (GLM procedure). In preacquisition and acquisition, stimulus (CS- and CS+) and phase (preacquisition, acquisition) were entered as within-subjects factors, and group (GAD and $\mathrm{HC}$ ) was entered as a between-subjects factor. Additionally, to assess differential dynamics during acquisition, a 2x3x2 ANOVA was conducted for each measure, with type of CS and Block (Acquisition 1, Acquisition 2, Acquisition 3) as within-subjects and Group as between-subjects factor. 
Generalization analyses included stimulus (CS-, class 1, class 2, class 3, class 4, and $\mathrm{CS}+)$ as within-subjects and group (GAD and HC) as between-subjects factor. All ANOVAs were followed by $t$-tests (and trend analyses in the case of generalization) when necessary. As in Lissek et al. (2010, 2013), quadratic trend analyses were specially important to test the shape of generalization gradients because it was expected that GAD participants (but not HC) would depart from the quadratic function found in healthy humans (see Lissek et al., 2010). Bivariate Pearson correlations were calculated to assess the relationship between GAD features (C-D scale and STAI-T scores) and our conditioned fear-generalization indexes. Between-groups differences in risk ratings and retrospective reports to the CS- were calculated with independent sample $t$-tests. Gender was excluded from the final analyses because it did not interact with the other factors, indicating that participants' gender did not affect conditioned fear-acquisition or generalization. EMG was analyzed using T-scores as well as raw data. Because similar results were obtained, only T-scores are presented. We report $\eta_{\mathrm{p}}{ }^{2}$ as a measure of effect size for main and interaction effects. Data were analyzed with SPSS 20. All analyses were conducted in GAD participants with no comorbidity $(\mathrm{n}=23)$ and repeated in GAD participants with comorbidity ( $\mathrm{n}=28)$.

\section{Results}

\section{Preliminary analyses}

As expected, GAD patients had statistically significant higher scores on the STAI-T, STAI-S, and ASI-3. GAD patients also showed higher ratings for the discomfort elicited by the startle probe than the HC. There were no differences between groups regarding awareness of the CS-US contingency, selected intensity of the US, and ratings of 
the discomfort produced by the shock (Table 1). In addition, groups did not differ in startle reactivity across the experiment, $F(3,153)=.57, p=.60, \eta_{\mathrm{p}}{ }^{2}=.01$, or in startle habituation before the experimental phases $F(8,336)=1.08, p=.37, \eta_{\mathrm{p}}{ }^{2}=.03$. The results of the these preliminary analyses were identical for GAD participants with comorbidity.

\section{Preacquisition and acquisition}

Risk ratings. A main effect of type of Stimulus, $F(1,49)=38.50, p<.001, \eta_{\mathrm{p}}{ }^{2}=.44$, a main effect of Group, $F(1,49)=3.90, p=.05, \eta_{\mathrm{p}}{ }^{2}=.07$, as well as a main effect of Phase $F(1,49)=79.64, p<.001, \eta_{\mathrm{p}}{ }^{2}=.61, \quad$ were found. Moreover, the Stimulus $x$ Phase interaction was significant, $F(1,49)=28.54, p<.001, \eta_{\mathrm{p}}{ }^{2}=.37$, driven by significantly increased risk ratings to the $\mathrm{CS}+$ versus $\mathrm{CS}$ - during the acquisition, $t(50)=8.30, p<.001$, but not during the preacquisition, $t(50)=1.83, p=.08$, indicating successful fear acquisition for the whole sample. However, neither the Stimulus x Group nor the Stimulus $\mathrm{x}$ Phase $\mathrm{x}$ Group interactions were significant, both $F \mathrm{~s}<1.22, p \mathrm{~s}>.28, \eta_{\mathrm{p}}{ }^{2}<.02$, showing that fear acquisition was similar in GAD and HC. Additionally, $t$-tests showed that risk ratings to the CS- during the acquisition phase were not significantly different between groups, $t(49)=1.01, p=.32$. Results were identical for GAD participants with comorbidity.

EMG. A main effect of type of Stimulus was found, $F(1,51)=32.44, p<.001, \eta_{\mathrm{p}}{ }^{2}=$ 39. Additionally, the Stimulus $x$ Phase interaction was significant $F(1,51)=4.47, p=.04$, $\eta_{\mathrm{p}}{ }^{2}=.08$, and was driven by significantly increased startle to the CS+ versus CS- during the acquisition, $t(52)=2.80, p<.001$, but not during the preacquisition phase, $t(52)=.63, p=$ 53. Similar to the Risk Ratings results, neither the Stimulus x Group nor the Stimulus $x$ Phase $\mathrm{x}$ Group interactions were significant, both $F \mathrm{~s}<.26, p \mathrm{~s}>.61, \eta_{\mathrm{p}}{ }^{2}<.02$, showing that 
fear acquisition was similar in GAD and HC. Results were identical for GAD participants with comorbidity.

The results of the ANOVA focusing on acquisition rate using the 3 acquisition blocks revealed increased risk ratings for the $\mathrm{CS}+$ versus the $\mathrm{CS}$ - for all 3 blocks and greater startle EMG for the CS+ versus the CS- in blocks 1 and 3 (see Table 2). The Stimulus $\times$ Group interaction was not significant, $F(1,100)=.09, p=.76, \eta_{\mathrm{p}}{ }^{2}=.01$, indicating that the "speed" of fear acquisition was similar in GAD and HC. Again, same results were obtained for GAD participants with comorbidity.

\section{Table 2.}

Means and standard deviations in the Preacquisition and Acquisition phases, for EMG and Risk Ratings by groups

\begin{tabular}{lcccccccc}
\hline \multirow{2}{*}{ PHASE } & \multicolumn{3}{c}{ Non-comorbid GAD participants } & \multicolumn{3}{c}{ Healthy controls } \\
& \multicolumn{2}{c}{ CS + CS } & \multicolumn{2}{c}{ CS- } & \multicolumn{2}{c}{ CS+ } & \\
& Mean & SD & Mean & SD & Mean & SD & Mean & SD \\
EMG & & & & & & & & \\
PRECON & 58.45 & 7.38 & 58.22 & 5.72 & 57.06 & 6.42 & 58.53 & 6.97 \\
& $(58.76)$ & $(7.79)$ & $(58.31)$ & $(5.44)$ & & & & \\
CON & 54.71 & 4.23 & 52.33 & 3.24 & 53.84 & 4.66 & 51.75 & 4.30 \\
& $(55.05)$ & $(4.57)$ & $(52.01)$ & $(3.11)$ & & & & \\
CON_1 & 54.61 & 6.58 & 53.12 & 5.20 & 56.11 & 7.28 & 53.27 & 6.47 \\
& $(55.04)$ & $(6.71)$ & $(53.26)$ & $(5.16)$ & & & & \\
CON_2 & 55.97 & 6.74 & 53.26 & 5.90 & 53.41 & 6.51 & 52.41 & 6.61 \\
& $(56.28)$ & $(6.92)$ & $(52.77)$ & $(5.78)$ & & & & \\
CON_3 & 53.31 & 8.11 & 50.38 & 5.76 & 51.83 & 6.09 & 49.58 & 6.10 \\
& $(53.59)$ & $(7.59)$ & $(49.93)$ & $(5.34)$ & & & & \\
RISK RATINGS & & & & & & & & \\
PRECON & 1.59 & 0.67 & 1.29 & 0.45 & 1.19 & 0.42 & 1.19 & 0.48 \\
& $(1.62)$ & $(0.65)$ & $(1.38)$ & $(0.55)$ & & & & \\
CON & 2.27 & 0.58 & 1.35 & 0.37 & 2.17 & 0.66 & 1.22 & 0.47 \\
& $(2.24)$ & $(0.59)$ & $(1.41)$ & $(0.49)$ & & & & \\
CON_1 & 1.97 & 0.45 & 1.33 & 0.58 & 1.63 & 0.58 & 1.23 & 0.55 \\
& $(1.95)$ & $(.48)$ & $(1.45)$ & $(0.65)$ & & & & \\
CON_2 & 2.38 & 0.77 & 1.45 & 0.61 & 2.25 & 0.75 & 1.23 & 0.57 \\
& $(2.39)$ & $(0.73)$ & $(1.45)$ & $(0.58)$ & & & & \\
CON_3 & 2.26 & 0.64 & 1.29 & 0.51 & 2.43 & 0.74 & 1.20 & 0.47 \\
& $(2.25)$ & $(.67)$ & $(1.35)$ & $(0.62)$ & & & & \\
\hline
\end{tabular}

PRECON; Preconditioning phase, CON; conditioning phase, CON_1; Block 1 of conditioning phase, CON_2; Block 2 of conditioning phase, CON_3; Block 3 of conditioning phase, SD; Standard Deviation. 
Retrospective Anxiety and Arousal. There was also evidence of fear conditioning for the whole sample in the retrospective self-reports of anxiety and arousal, as shown by a main effect of type of Stimulus, both $F \mathrm{~s}>64.02, p \mathrm{~s}<.001, \eta_{\mathrm{p}}{ }^{2}>.55$, driven by greater reported anxiety and arousal to the CS+ compared to the CS- in both GAD (both $p s<$ $.001)$ and $\mathrm{HC}$ (both $p \mathrm{~s}<\quad .001)$. The Stimulus $\times$ Group interaction was not significant, both $F \mathrm{~s}<.74, p s>.34, \eta_{\mathrm{p}}^{2}<.01$, indicating that these differences were similar across groups. Although this interaction was not significant, $t$-tests showed between-group differences in the retrospective ratings of the CS-, with GAD patients showing higher anxiety $(\mathrm{M}=3.18, \mathrm{SD}=2.12 \mathrm{vs} \mathrm{M}=1.40, \mathrm{SD}=1.40)$ and $\operatorname{arousal}(\mathrm{M}=4.04, \mathrm{SD}=2.61$ vs $\mathrm{M}=2.43, \mathrm{SD}=2.05)$ than $\mathrm{HC}$, both $t \mathrm{~s}(50)>2.50$, all $p \mathrm{~s}<.01$. Identical results were obtained for GAD participants with comorbidity.

\section{Conditioned fear-generalization}

Risk ratings. Generalization of fear conditioning was evidenced by main effects of Stimulus type in both GAD, $\mathrm{F}(5,100)=17.61, \mathrm{p}<.001, \eta \mathrm{p} 2=.46$, and $\mathrm{HC}, \mathrm{F}(5,140)=$ $41.54, \mathrm{p}<.001, \eta \mathrm{p} 2=.60$ indicating that participants responses varied as a function of the stimuli presented. Comparison of risk ratings to each stimulus with ratings to the CS- ( $\alpha$ set at $\mathrm{p}<.01$ ) within each group revealed that GAD participants showed greater risk ratings to the CS+ than to the CS-, $\mathrm{t}(20)=6.26, \mathrm{p}<.001$ as well as greater risk ratings to all GSs in comparison to the CS-, all ts $(2)>2.46$, ps $<.02$. A similar pattern was observed for HC, with greater risk ratings to the $\mathrm{CS}+$ than to the $\mathrm{CS}-, \mathrm{t}(28)=7.64, \mathrm{p}<.001$, and with greater ratings to class 4,3 and $2 \mathrm{GSs}$; all ts $(28)>1.54$, ps $<.00$. Only ratings to class $1 \mathrm{GSs}$ did 
not differ from ratings to CS-, $\operatorname{ts}(28)=1.54$, $\mathrm{ps}=.13$. Generalization of risk ratings therefore extended to all GSs in GAD participants and to all but one GSs in HC. However, the main effect of Group, $F(1,48)=.12, p=.73, \eta p 2=.00$ and the Stimulus X Group interaction were not significant, $\mathrm{F}(5,240)=2.00, \mathrm{p}=.14, \eta \mathrm{p} 2=.04$ (Figure 1). Moreover, independent sample t-tests showed that risk ratings to the CS- during the generalization phase were similar across groups, $\mathrm{t}(48)=.88, \mathrm{p}=.38$.

Analyses for GAD participants with comorbidity were almost identical except for the Stimulus X Group interaction that in this case was significant: $F(5,255)=2.87, p=.01$, $\eta \mathrm{p} 2=.05$. However, this was not attributable to between group-differences in the quadratic component of the generalization gradients of the groups (the Stimulus $\times$ Group quadratic trend was not significant; $F(1,51)=1.83, \mathrm{p}=.18)$ but rather to the fact that GAD patients with comorbidity reported higher risk to class $1 \mathrm{GS}$ than $\mathrm{HC}, \mathrm{t}(52)=2.21, \mathrm{p}=.02$.

\section{Figure 1.}

Generalization gradients by group. Bars represent standard errors of the mean.

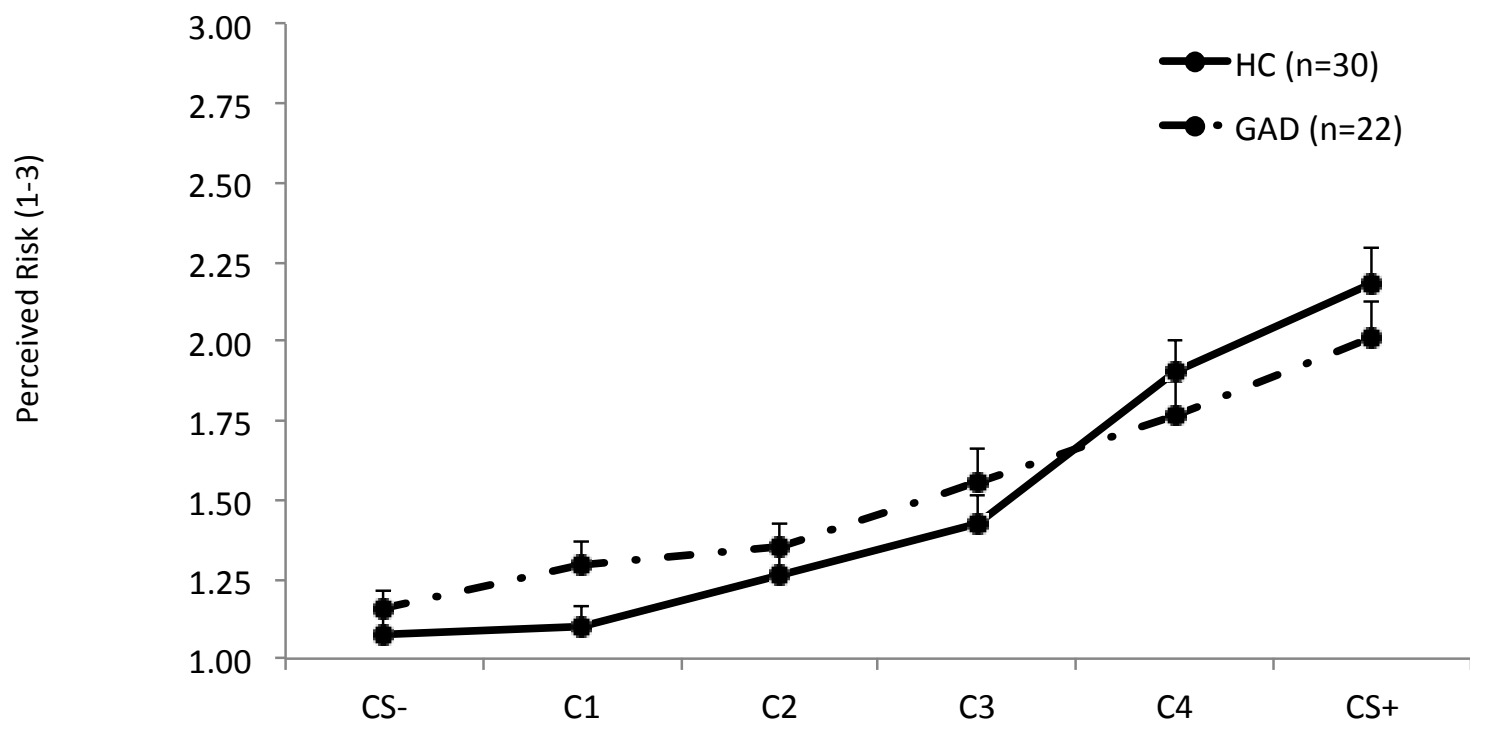


EMG. For the whole sample, a main effect of type of Stimulus was found, F(5, 255) $=13.19, \mathrm{p}<.001, \eta \mathrm{p} 2=.21$. Generalization of startle responses across stimuli was analyzed by comparing responses elicited during the presentation of the CS- with responses elicited during each GSs for each group, with $\alpha$ adjusted by means of a Bonferroni correction ( $\mathrm{p}<$ $.05 / 5=.01)$. Paired-samples $t$ tests in GAD subjects showed that startle responses elicited during the presentation of the CS+ were significantly higher than responses elicited during the CS-, $\mathrm{t}(22)=3.28, \mathrm{ps}=.00$. Additionally, responses elicited during the class 4 and class 3 GSs were also greater than those elicited during the CS-, both ts $(22)>3.86$, ps $<.001$. In contrast, responses elicited during the presentation of the class 2 and class 1 GSs did not differ from responses elicited during the CS-, both ts $(22)<1.25$, ps $>.05$. Paired-samples $t$ tests in $\mathrm{HC}$ showed the same pattern. Startle responses elicited during the presentation of the CS+ were significantly higher than responses elicited during the CS-, $t(29)=2.62$, ps $=$ .01. Additionally, responses elicited during the class 4 and class 3 GSs were also greater than those elicited during the CS-, both ts $(29)>1.99$, ps $<.05$. Only responses elicited during the presentation of the class 2 and class 1 GSs did not differ from responses elicited during the CS-, both ts $(29)<.95$, ps $>.05$. Thus, conditioned fear, as measured by startle potentiation, extended to the class 4 and class 3 GSs. However, the generalization gradient did not differ across groups, as indicated by a non-significant Stimulus $\times$ Group interaction $\mathrm{F}(5,255)=.88, \mathrm{p}>.05, \eta \mathrm{p} 2=.02$ (Figure 2$)$. Identical results were obtained for the GAD group with comorbidity. 


\section{Figure 2.}

Generalization gradients by group. Bars represent standard errors of the mean.

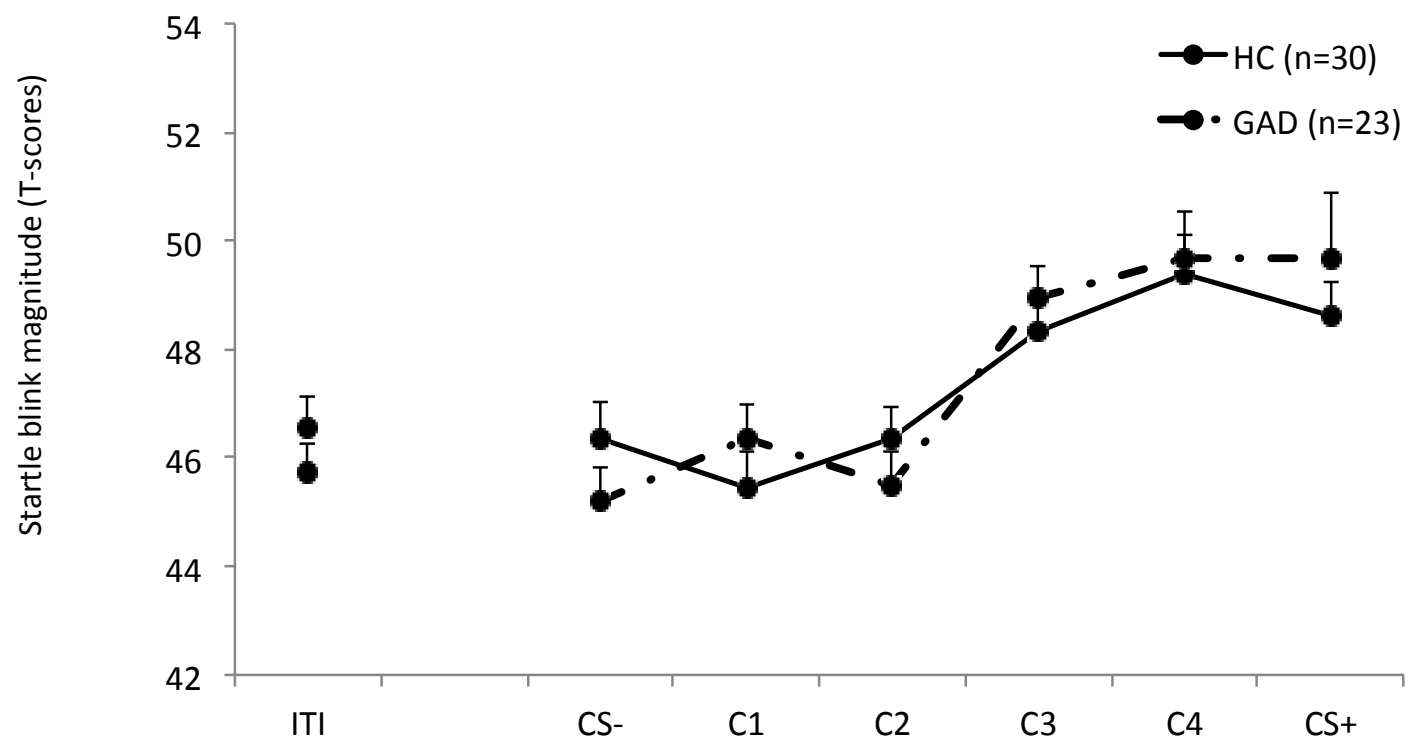

Following Lissek et al. (2013), the shape of generalization gradients was also assessed by calculating the degree to which each gradient departed from linearity using the equation: linear departure $=([\mathrm{CS}++\mathrm{CS}-] / 2)-([\mathrm{GS} 1+\mathrm{GS} 2+\mathrm{GS} 3+\mathrm{GS} 4] / 4)$. Risk ratings results indicated that linear departures for GAD participants $(M=.20, S D=.26)$ and $\mathrm{HC}(\mathrm{M}=.09, \mathrm{SD}=.31)$ were not significantly different, $t(51)=1.32, \mathrm{p}=.19$. Standardized startle data were similar with GAD participants $(\mathrm{M}=-1.77, \mathrm{SD}=3.44)$ and $\mathrm{HC}(\mathrm{M}=.12, \mathrm{SD}=3.16)$ not being significantly different, $t(52)=.33, \mathrm{p}=.75$. Both measures were not either significantly different for GAD participants with comorbidity.

\section{Retrospective Anxiety and Arousal.}


Overall self-reported anxiety and arousal following the generalization phase revealed a main effect of type of Stimulus, both $F_{\mathrm{s}}>53.98, p \mathrm{~s}<.001, \eta_{\mathrm{p}}{ }^{2}>.51$, driven by greater reported anxiety and arousal to the CS+ compared to the CS- in both GAD (both $p \mathrm{~s}$ $<.001$ ) and HC (both $p s<\quad .001$ ). Similar to the acquisition phase, the Stimulus $\times$ Group interaction was not significant, both $F \mathrm{~s}<.74, p>.34, \eta_{\mathrm{p}}{ }^{2}=.01$. Although this interaction was not significant, $t$-tests showed between-group differences in the retrospective ratings of the CS-, with GAD patients showing higher anxiety $(\mathrm{M}=2.81, \mathrm{SD}=2.08$ vs $\mathrm{M}=1.57$, $\mathrm{SD}=1.38$ respectively $)$ and arousal $(\mathrm{M}=3.18, \mathrm{SD}=2.20$ vs $\mathrm{M}=1.80, \mathrm{SD}=1.69$ respectively) than $\mathrm{HC}$, both $t \mathrm{~s}(50)>2.60$, all $p \mathrm{~s}<.03$. Results for GAD participants with comorbidity were almost identical, with a non-significant Stimulus $\times$ Group interaction (both $F \mathrm{~s}<.58, p>.45, \eta_{\mathrm{p}}{ }^{2}=.01$ ) but $t$-tests showing between-group differences in the retrospective ratings of the CS-and GAD participants showing higher anxiety $(\mathrm{M}=2.83$, $\mathrm{SD}=2.24$ vs $\mathrm{M}=1.57, \mathrm{SD}=1.38$ respectively $)$ and arousal $(\mathrm{M}=3.12, \mathrm{SD}=2.49$ vs $\mathrm{M}=$ $1.80, \mathrm{SD}=1.69$ respectively) than $\mathrm{HC}$, both $t \mathrm{~s}(52)>2.32$, all $p \mathrm{~s}<.02$.

\section{Complementary analyses}

Given the significant difference between groups, we repeated our analyses using the variable 'startle probe discomfort' as a covariate, but results (using both GAD participants with and without comorbidity) remained unchanged (data not shown but analyses available upon request).

The difference in the risk ratings to the CS+ and to the CS- in the preacquisition phase approached significance $(p=.08)$. Given this unexpected finding, we repeated our analyses excluding those subjects whose difference score (CS+ - CS-) during preacquisition 
was 0.5 points above or below $0(\mathrm{GAD}, \mathrm{n}=6 ; \mathrm{HC}, \mathrm{n}=2)$. This rendered the Stimulus $\mathrm{x}$ Group interaction during preacquisition non-significant $(p=.97)$. After this, acquisition and generalization results remained unchanged. We also compared the number of participants for each group that received the largest or smallest ring as CS+. This proportion was not different across groups $(\mathrm{GAD}$ : largest ring $=10 ;$ smallest ring $=12 ; \mathrm{HC}$ : largest ring $=16$; smallest ring $\left.=14 ; \chi^{2}=.32, p=.58\right)$. The same set of analyses using GAD participants with comorbidity yielded almost identical results, except for the Stimulus X Group interaction during generalization, that now became non-significant $(\mathrm{p}=.10)$.

\section{Discussion}

In the present study, we aimed to examine fear acquisition and generalization in GAD patients using a fear conditioning paradigm assessing FPS and subjective responses. As expected, GAD patients and controls did not differ as regards fear acquisition. However, contrary to our hypothesis, both groups did not differ in most indexes of conditioned fear-generalization.

There are very few studies that have investigated fear acquisition in GAD using a fear conditioning paradigm and are comparable to ours. The study by Thayer et al. (2000) mentioned in the introduction used a higher-order conditioning procedure. An earlier study by Pitman and Orr (1986) found no differences in differential conditioning during acquisition between GAD patients (as defined by DSM-III criteria) and controls, but comparison with this study is difficult given the changes in GAD criteria from DSM-III-R onwards. These changes emphasized the concept of GAD as a separate disorder, its duration/persistence (change from 1 to 6 months) and focused on the role of worry. Therefore only the study by Lissek et al. (2013) has investigated fear conditioning in GAD 
as it is currently defined in the DSM-IV-TR (APA, 2000) or DSM-V (APA, 2013) and using a similar paradigm.

In terms of acquisition, our results agree with those of Lissek et al. (2013) and indicate that GAD individuals have "normal" fear acquisition. Some previous differential conditioning studies in PD (Lissek et al., 2009) or PTSD (Jovanovic et al., 2009, 2010) suggest that these patients have difficulties in suppressing fear responses to the CS- (i.e., deficits in inhibition learning) but this question has not specifically been investigated in GAD (see Lissek et al., 2005). In any case, it must be noted that most previous studies conducted in this area do not provide a direct test of differences in inhibition learning (see Mineka \& Oehlberg, 2008). Recently, new experimental methods have been developed (Jovanovic et al., 2005; Kindt \& Soeter, 2014), which can assess the independent contributions of fear excitation and fear inhibition, although, to our knowledge, they have not been tested in GAD.

The present results on fear acquisition are also in agreement with studies that have found that (non-clinical) individuals with high trait anxiety show similar fear acquisition than those with low trait-anxiety (Fredrikson \& Georgiades, 1992; Otto et al., 2007; Pineles, Vogt, \& Orr, 2009; Torrents-Rodas et al., 2013). Nevertheless, as stated in the introduction, results are not conclusive (see Baas, van Ooijen, Goudriaan, \& Kenemans 2008; Indovina et al., 2011; Gazendam, Kamphuis, \& Kindt, 2013). Recent research suggests that complex fear conditioning paradigms (e.g. the blocking and the protection-from-overshadowing procedure) may be better suited than the "typical" differential fear conditioning paradigm to detect individual differences variables involved in fear conditioning (Arnaudova et al., 2013; see Beckers et al., 2013). 
The hypothesized greater conditioned fear-generalization in patients as compared to controls was overall not confirmed. The only signs of increased conditioned fear-generalization emerged for retrospective ratings of the CS-, that were (or tended to be) higher among GAD participants and for the risk ratings to the generalization stimuli, that suggested also a slightly higher tendency to generalize in GAD. This is surprising because we used the same paradigm as Lissek et al. (2013). Apart from small socio-demographic differences (our sample was slightly younger; $M=24$ versus $M=31$ in Lissek et al. and $100 \%$ Caucasian versus $73 \%$ in Lissek et al.), there seem to be few differences between Lissek et al. study (2013) and ours. The main difference is that half of the patients in Lissek et al. (2013) had a comorbid anxiety disorder. Also, our ratings of discomfort for the startle probe were higher for the GAD than for the $\mathrm{HC}$ group (this information is not reported in Lissek et al. study), but controlling for this variable did not change our results. It could also be argued that the discomfort ratings for the US in our study are relatively low, but they were similar in both groups, and this should have affected (and it did not) fear acquisition. As in Lissek et al. (2013), we did not find between-group differences during acquisition. This is important given the effects that differential fear acquisition may have on conditioned fear-generalization (see Dunsmoor, Mitroff, \& LaBar, 2009).

Our generalization results are in line with another recent study assessing conditioned fear-generalization in GAD (Greenberg et al., 2013). In this study, conditioned fear-generalization gradients -as assessed with pupillary responses or risk ratings ${ }^{3}$ - were similar for GAD patients and healthy controls. This was an instructed fear study, but results on conditioned fear-generalization seem to be comparable whether participants are informed on the CS-US contingencies or learn them through direct experience (see 
Greenberg et al., 2013). It must be noted, however, that $50 \%$ of GAD patients in this study had comorbid major depression. The current results are also in agreement with a previous non-clinical study from our group, where conditioned fear-generalization gradients were compared among individuals with different levels of trait anxiety (as measured with the STAI-T) and no differences emerged (Torrent-Rodas et al., 2013).

If the results of our study were replicated, we would then need to answer the question: Why are GAD patients not characterized by enhanced fear generalization?

First, it is important to note that conditioned fear-generalization may occur along different dimensions. In the current work (as well as in Lissek et al., 2013 and Greenberg et al., 2013 studies) the focus was on conditioned fear-generalization based on a dimension of physical similarity. However, generalization may also occur along a dimension of fear intensity. In fact, a recent study suggests that generalization of conditioned fear in humans is determined by fear intensity rather than physical similarity (Dunsmoor, Mitroff, \& LaBar, 2009). It could be the case that GAD (or other anxiety disorders) is characterized by abnormalities in conditioned fear-generalization determined by the former rather than the latter. Similarly, research on conditioned fear-generalization in humans so far has mainly focused on dimensional stimulus changes, but it has been shown that discrete feature changes may also influence generalization (Vervliet \& Geens, 2013). To what extent these two mechanisms (generalization based on fear intensity or feature learning) are involved in anxiety disorders remains unknown.

Also, most previous studies in anxiety disorders have focused on cued conditioned fear-generalization and very little research has been conducted on context fear generalization. This is especially important in GAD (see below) because chronic anxiety 
seems to be better modeled as the result from context rather than cue conditioning (Davis, 1998; Luyten, Vansteenwegen, van Kuyck, Gabriëls, \& Nuttin, 2011).

Second, it could be the case that conditioned fear-generalization abnormalities characterize some anxiety disorders but not others. So far, evidence for abnormal conditioned fear-generalization has been mainly found in PD (Lissek et al., 2010). Although both PD and GAD are currently classified as anxiety disorders, they differ in many aspects. Panic disorder involves fear of specific stimuli (for example, somatic experiences), whereas GAD is characterized by more diffuse anxiety. More importantly, whereas high arousal could be associated with $\mathrm{PD}$, decreased arousal seems to characterize GAD (Hoehn-Saric, McLeod, \& Zimmerli, 1989; Hoehn-Saric, McLeod, Funderburk, \& Kowalski, 2004). Previous studies suggest that fear sensitization (i.e., an enhanced fear response after experiencing intense fear) plays an important role in conditioned fear-generalization (Dunsmoor, Mitroff, \& LaBar, 2009). Given that increases in general arousal may sensitize some fear-relevant stimuli (see, for example, Öhman \& Mineka, 2001), it is therefore possible that only anxiety disorders characterized by increased arousal show augmented conditioned fear-generalization.

Third, as stated in the introduction, the emphasis of current models of GAD is not on variables related to fear acquisition/generalization. Other mechanisms, especially those related to the avoidance of internal affective experiences, may play a greater role in the etiology of GAD (see Borkovec, Alcaine, \& Behar, 2004; Behar et al., 2009).

We tested acquisition and generalization effects in GAD patients with and without anxious comorbidity but the results were almost identical. It may be argued that testing GAD participants with no comorbidity limits the generalizability of our findings because comorbidity is the rule rather than the exception in GAD. However, it is important 
to note that GAD is not "more comorbid" than other anxiety disorders, at least at an epidemiological level (Kessler, 2000; Kessler, Keller, \& Wittchen, 2001). The high comorbidity seen in clinical samples in GAD is an artifact of treatment-sample selection rather than inherent to the disorder (Wittchen, 2002; Kessler, 2000). Moreover, a better understanding of non-comorbid GAD is important because it is associated with significant impairment in both adult (Bruce, Machan, Dyck, \& Keller, 2001) and pediatric (Alfano, 2012) samples. On the other hand, the fact that we repeated our analyses GAD participant with comorbidity provides a unique perspective on the possible effects of comorbidity on fear conditioning abnormalities, a topic which has received little attention so far. If we assume that comorbidity is an index of severity (i.e, that patients with comorbidity are generally more severe than non-comorbid ones), one interesting possibility is that these abnormalities may be significantly associated with severity (current symptom) rather than the presence/absence of the disorder, as seen in a recent study in PTSD patients (Jovanovic et al., 2010). Moreover, this would be in agreement with data showing that fear learning abnormalities are also present in other "non-anxiety" disorders such as schizophrenia (see Holt, Coombs, Zeidan, Goff, \& Milad, 2012). Although our results suggest that anxious comorbidity does not make a significant difference, it is still possible that "other comorbidities" have an effect on variables related to fear learning. In this repect, a limitation of our study is that we did not measure depressive symptoms, which may influence fear conditioning processes (Dibbets, van den Broek, \& Evers, 2014). Another limitation is aht other dimensional instruments for GAD are available (e.g. the Penn-State Worry Questionnaire; Meyer, Miller, Metzger, \& Borkovec, 1990) which probably tap better into the GAD construct and which should be used in future investigations. 
To summarize, we did not find that overall GAD patients differ from healthy controls in conditioned fear acquisition or generalization. Although great advances have been made to study etiological accounts on GAD, much is still unclear. Future investigations should address associative as well as non-associative processes involved in the disorder. On a broader scale, further research is needed on the role of comorbidity in fear learning abnormalities in anxiety disorders. 


\section{References}

Alfano, C. A. (2012). Are children with "pure" generalized anxiety disorder impaired? A comparison with comorbid and healthy children. Journal of Clinical Child and Adolescent Psychology, 41, 739-745.

American Psychiatric Association. (2000). Diagnostic and statistical manual of mental disorders (4th ed., text rev. ed.). Washington, DC: American Psychiatric Press. American Psychiatric Association. (2013). Diagnostic and statistical manual of mental disorders (5th ed.). Washington, DC: American Psychiatric Press.

Arnaudova, I., Krypotos, A. M., Effting, M., Boddez, Y., Kindt, M., \& Beckers, T. (2013). Individual differences in discriminatory fear learning under conditions of ambiguity: a vulnerability factor for anxiety disorders? Frontiers in Psychology, 4, $1-9$.

Baas, J. M. P., van Ooijen, L., Goudriaan, A., \& Kenemans, J. L. (2008). Failure to condition to a cue is associated with sustained contextual fear. Acta Psychologica, $127,581-592$

Beckers, T., Krypotos, A. M., Boddez, Y., Effting, M., \& Kindt, M. (2013). What's wrong with fear conditioning? Biological Psychology, 92, 90-96.

Behar, E., DiMarco, I. D., Hekler, E. B., Mohlman, J., \& Staples, A. M. (2009). Current theoretical models of generalized anxiety disorder (GAD): Conceptual review and treatment implications. Journal of Anxiety Disorders, 23, 1011-1023.

Blumenthal, T. D., Cuthbert, B. N., Filion, D. L., Hackley, S., Lipp, O. V., \& Van Boxtel, A. (2005). Committee report: Guidelines for human startle eyeblink electromyographic studies. Psychophysiology, 42, 1-15. 
Bobes, J. (1998). A Spanish validation study of the MINI international neuropsychiatric interview.European Psychiatry, 13, 198-199.

Bobes, J., García-Calvo, C., Prieto, R., García-García, M., \& Rico-Villademoros, F. (2006).Propiedades psicométricas de la versión española de la Escala de Detección del Trastorno de Ansiedad Generalizada según DSM-IV de Carroll y Davidson.[Psychometric properties of the Spanish version of the Generalized Anxiety Disorder Screening Scale according to DSM-IV of Carroll and Davidson].Actas Españolas de Psiquiatría, 34, 83-93.

Borkovec, T. D., Alcaine, O., \& Behar, E. (2004). Avoidance theory of worry and generalized anxiety disorder. In R. G. Heimberg, C. L. Turk, \& D. S. Mennin (Eds.), Generalized anxiety disorder: Advances in research and practice (pp. 77-108). New York: Guilford Press.

Bruce, S. E., Machan, J. T., Dyck, I., \& Keller, M. B. (2001). Infrequency of "pure” GAD: impact of psychiatric comorbidity on clinical course. Depression and Anxiety, 14, 219-225.

Davis, M. (1998). Are different parts of the extended amygdala involved in fear versus anxiety? Biological Psychiatry, 44, 1239-1247.

Dawson, M. E., \& Reardon, P. (1973). Construct validity of recall and recognition postconditioning measures of awareness. Journal of Experimental Psychology, 98, $308-315$.

Dibbets, P., van den Broek, A., \& Evers, E. A. (2014). Fear conditioning and extinction in anxiety-and depression-prone persons. Memory.

DOI:10.1080/09658211.2014.886704 
Dunsmoor, J. E., Mitroff, S. R., \& LaBar, K. S. (2009). Generalization of conditioned fear along a dimension of increasing fear intensity.Learning \& Memory, 16, 460-469.

Fisher, P. L., \& Durham, R. C. (1999). Recovery rates in generalized anxiety disorder following psychological therapy: an analysis of clinically significant change in the STAI-T across outcome studies since 1990. Psychological Medicine, 29, $1425-1434$.

Fredrikson, M., \& Georgiades, A. (1992).Personality dimensions and classical conditioning of autonomic nervous system reactions.Personality and Individual Differences, 13, 1013-1020.

Gazendam, F. J., Kamphuis, J. H., \& Kindt, M. (2013). Deficient safety learning characterizes high trait anxious individuals. Biological Pychology, 92, 342-352.

Graham, B. M., \& Milad, M. R. (2011). The study of fear extinction: implications for anxiety disorders. American Journal of Psychiatry, 168, 1255-1265.

Greenberg, T., Carlson, J. M., Cha, J., Hajcak, G., \& Mujica Parodi, L. R. (2012). Ventromedial prefrontal cortex reactivity is altered in generalized anxiety disorder during fear generalization. Depression and Anxiety, 30, 242-250.

Hajcak, G., Castille, C., Olvet, D. M., Dunning, J. P., Roohi, J., \& Hatchwell, E. (2009).Genetic variation in brain derived neurotrophic factor and human fear conditioning.Genes, Brain and Behavior, 8, 80-85.

Hermann, C., Ziegler, S., Birbaumer, N., \& Flor, H. (2002). Psychophysiological and subjective indicators of aversive Pavlovian conditioning in generalized social phobia.Biological Psychiatry, 52, 328-337.

Hoehn-Saric, R., McLeod, D. R., \& Zimmerli, W. D. (1989). Somatic manifestations in women with generalized anxiety disorder: Psychophysiological responses to 
psychological stress. Archives of General Psychiatry, 46, 1113-1119.

Hoehn-Saric, R., McLeod, D. R., Funderburk, F., \& Kowalski, P. (2004). Somatic Symptoms and Physiologic Responses in Generalized Anxiety Disorder and Panic Disorder: An Ambulatory Monitor Study. Archives of General Psychiatry, 61, 913-921.

Holt, D.J., Coombs, G., Zeidan, M.A., Goff, D.C., \& Milad, M.R. (2012). Failure of neural responses to safety cues in schizophrenia. Archives of General Psychiatry, 69, 893-903.

Indovina, I., Robbins, T. W., Núñez-Elizalde, A. O., Dunn, B. D., \& Bishop, S. J. (2011). Fear-conditioning mechanisms associated with trait vulnerability to anxiety in humans. Neuron, 69, 563-571.

Joos, E., Vansteenwegen, D., \& Hermans, D. (2012). Worry as a predictor of fear acquisition in a nonclinical sample. Behavior Modification, 36, 723-750.

Jovanovic, T., Keyes, M., Fiallos, A., Myers, K. M., Davis, M., \& Duncan, E. J. (2005). Fear potentiation and fear inhibition in a human fear-potentiated startle paradigm. Biological Psychiatry, 57, 1559-1564.

Jovanovic, T., Norrholm, S. D., Fennell, J. E., Keyes, M., Fiallos, A. M., Myers, K. M., et al. (2009). Posttraumatic stress disorder may be associated with impaired fear inhibition: Relation to symptom severity.Psychiatry Research, 167, 151-160.

Jovanovic, T., Norrholm, S. D., Blanding, N. Q., Davis, M., Duncan, E., Bradley, B., et al. (2010). Impaired fear inhibition is a biomarker of PTSD but not depression.Depression and Anxiety, 27, 244-251. 
Kessler, R. C. (2000). The epidemiology of pure and comorbid generalized anxiety disorder: a review and evaluation of recent research. Acta Psychiatrica Scandinavica, 10, 27-13.

Kessler, R. C., Keller, M. B., \& Wittchen, H. U. (2001). The epidemiology of generalized anxiety disorder. Psychiatric Clinics of North America, 24, 19-39.

Kindt, M., \& Soeter, M. (2014). Fear Inhibition in High Trait Anxiety. PloS One, 9, 1-6.

Lecrubier, Y., Sheehan, D. V., Weiller, E., Amorim, P., Bonora, I., Sheehan, K. H., Janavs, J., \& Dunbar, G. C. (1997). The Mini International Neuropsychiatric Interview (MINI). A short diagnostic structured interview: reliability and validity according to the CIDI. European Psychiatry, 12, 224-231.

Lissek, S., Powers, A. S., McClure, E. B., Phelps, E. A., Woldehawariat, G., Grillon, C., \& Pine, D. S. (2005). Classical fear conditioning in the anxiety disorders: A meta-analysis. Behaviour Research and Therapy, 43, 1391-1424.

Lissek, S., Levenson, J., Biggs, A. L., Johnson, L. L., Ameli, R., Pine, D. S., \& Grillon, C. (2008a). Elevated fear conditioning to socially relevant unconditioned stimuli in social anxiety disorder. American Journal of Psychiatry, 165, 124-132.

Lissek, S., Biggs, A. L., Rabin, S. J., Cornwell, B. R., Alvarez, R. P., Pine, D. S., \& Grillon, C. (2008b). Generalization of conditioned fear-potentiated startle in humans: experimental validation and clinical relevance. Behaviour Research and Therapy, 46, 678-687.

Lissek, S., Rabin, S. J., McDowell, D. J., Dvir, S., Bradford, D. E., Geraci, M., Pine, D. S., \& Grillon, C. (2009). Impaired discriminative fear-conditioning resulting from elevated fear responding to learned safety cues among individuals with panic disorder. Behaviour Research and Therapy, 47, 111-118. 
Lissek, S., Rabin, S., Heller, R. E., Lukenbaugh, D., Geraci, M., Pine, D. S., \& Grillon, C. (2010). Overgeneralization of conditioned fear as a pathogenic marker of panic disorder. The American Journal of Psychiatry, 167, 47-55.

Lissek, S. (2012). Toward an account of clinical anxiety predicated on basic, neurally mapped mechanisms of pavlovian fear-learning: the case for conditioned overgeneralization. Depression and Anxiety, 29, 257-263.

Lissek, S., Kaczkurkin, A. N., Rabin, S., Geraci, M., Pine, D. S., \& Grillon, C. (2013). Generalized Anxiety Disorder Is Associated with Overgeneralization of Classically Conditioned Fear. Biological Psychiatry, 75, 909-915.

Luyten, L., Vansteenwegen, D., van Kuyck, K., Gabriëls, L., \& Nuttin, B. (2011). Contextual conditioning in rats as an animal model for generalized anxiety disorder.Cognitive, Affective, \& Behavioral Neuroscience, 11, 228-244.

Meyer, T.J., Miller, M.L., Metzger, R.L., Borkovec, T.D. (1990). Development and validation of the Penn State Worry Questionnaire.Behaviour Research and Therapy, $28,487-495$.

Michael, T., Blechert, J., Vriends, N., Margraf, J., \& Wilhelm, F. H. (2007). Fear conditioning in panic disorder: Enhanced resistance to extinction. Journal of Abnormal Psychology, 116, 612-617.

Milad, M. R., Orr, S. P., Lasko, N. B., Chang, Y., Rauch, S. L., \& Pitman, R. K. (2008). Presence and acquired origin of reduced recall for fear extinction in PTSD: results of a twin study. Journal of Psychiatric Research, 42, 515-520.

Milad, M. R., Furtak, S. C., Greenberg, J. L., Keshaviah, A., Im, J. J., Falkenstein, M. J., Wilhelm, S., et al. (2013). Deficits in conditioned fear extinction in obsessive-compulsive disorder and neurobiological changes in the fear circuit. 
JAMA Psychiatry, 70, 608-618.

Mineka, S., \& Oehlberg, K. (2008).The relevance of recent developments in classical conditioning to understanding the etiology and maintenance of anxiety disorders.Acta Psychologica, 127, 567-580.

Mineka, S., \& Zinbarg, R. (2006). A contemporary learning theory perspective on the etiology of anxiety disorders: It's not what you thought it was. American Psychologist, 61, 10-26.

Newman, M. G., Llera, S. J., Erickson, T. M., Przeworski, A., \& Castonguay, L. G. (2013). Worry and Generalized Anxiety Disorder: A Review and Theoretical Synthesis of Evidence on Nature, Etiology, Mechanisms, and Treatment. Annual Review of Clinical Psychology, 9, 275-297.

Öhman, A., \& Mineka, S. (2001). Fears, phobias, and preparedness: toward an evolved module of fear and fear learning. Psychological Review, 108, 483-552.

Orr, S. P., Metzger, L. J., Lasko, N. B., Macklin, M. L., Peri, T., \& Pitman, R. K. (2000). De novo conditioning in trauma-exposed individuals with and without posttraumatic stress disorder.Journal of Abnormal Psychology, 109, 290-299.

Otto, M. W., Leyro, T. M., Christian, K., Deveney, C. M., Reese, H., Pollack, M. H.,\& Orr, S. P. (2007). Prediction of "fear" acquisition in healthy control participants in a de novo fear-conditioning paradigm.Behavior Modification, 31, 32-51.

Otto, M. W., Moshier, S. J., Kinner, D. G., Simon, N. M., Pollack, M. H., \& Orr, S. P. (2014). De Novo Fear Conditioning Across Diagnostic Groups in the Affective Disorders: Evidence for Learning Impairments. Behavior Therapy, 45, 619-629. 
Peri, T., Ben-Shakhar, G., Orr, S. P., \& Shalev, A. Y. (2000).Psychophysiologic assessment of aversive conditioning in posttraumatic stress disorder.Biological Psychiatry, 47, $512-519$

Pineles, S. L., Vogt, D. S., \& Orr, S. P. (2009). Personality and fear responses during conditioning: Beyond extraversion. Personality and Individual Differences, 46, 43-53.

Pitman, R. K., \& Orr, S. P. (1986). Test of the conditioning model of neurosis: Differential aversive conditioning of angry and neutral facial expressions in anxiety disorder patients. Journal of Abnormal Psychology, 95, 208-213.

Sandin, B., Valiente, R. M., Chorot, P., \& Santed, M. A. (2007). ASI-3: nueva escala para la evaluación de la sensibilidad a la ansiedad. [ASI-3: new scale for the assessment of anxiety sensitivity]. Revista de Psicopatología y Psicología Clínica, 12, 91-104.

Sheehan, D. V., Lecrubier, Y., Sheehan, K. H., Amorim, P., Janavs, J., Weiller, E., Herqueta, T., Baker, R., \& Dunbar, G. C. (1998). The Mini-International Neuropsychiatric Interview (M.I.N.I.): The development and validation of a structured diagnostic psychiatric interview for DSM-IV and ICD-10. Journal of Clinical Psychiatry, 59, 22-33.

Spielberger, C. D., Gorsuch, R. R., \& Lushene, R. E. (1982). STAI. Cuestionario de ansiedad Estado/Rasgo (1st ed.).[Validation of the Spanish version of the State Trait Anxiety Scale (STAI)]. Madrid: Técnicos Especialistas Asociados (TEA).

Thayer, J. F., Friedman, B. H., Borkovec, T. D., Johnsen, B. H., \& Molina, S. (2000). Phasic heart period reactions to cued threat and nonthreat stimuli in generalized anxiety disorder. Psychophysiology, 37, 361-368. 
Torrents-Rodas, D., Fullana, M. A., Bonillo, A., Caseras, X., Andión, Ó., \& Torrubia, R. (2013). No effect of trait anxiety on differential fear conditioning or fear generalization. Biological Psychology, 92, 185-190.

Tinoco-González, D., Fullana, M. A., Torrents-Rodas, D., Bonillo, A., Vervliet, B., Pailhez, G., Farré, M., Pérez, V., \& Torrubia, R. (2014). Conditioned Subjective Responses to Socially Relevant Stimuli in Social Anxiety Disorder and Subclinical Social Anxiety. Clinical Psychology \& Psychotherapy. DOI: 10.1002/cpp.1883

Vervliet, B., \& Geens, M. (2014). Fear generalization in humans: impact of feature learning on conditioning and extinction. Neurobiology of Learning and Memory, 113, $143-148$.

Vervliet, B., Vansteenwegen, D., \& Eelen, P. (2004). Generalization of extinguished skin conductance responding in human fear conditioning. Learning and Memory, 11, $555-558$

Vervliet, B., Vansteenwegen, D., Baeyens, F., Hermans, D., \& Eelen, P. (2005). Return of fear in a human differential conditioning paradigm caused by a stimulus change after extinction. Behaviour Research and Therapy, 43, 357-371.

Wittchen, H. U. (2002). Generalized anxiety disorder: prevalence, burden, and cost to society. Depression and Anxiety, 16, 162-171. 